\title{
Progress and Prospects of the FTU Collective Thomson Scattering Diagnostics
}

\author{
William Bin*; Alessandro Bruschi, Lorenzo Figini, Saul Garavaglia, Gustavo \\ Granucci, Giovanni Grosso, Vittoria Mellera, Daniele Minelli, Alessandro Moro, \\ Antonio Nardone, Alessandro Simonetto, Umberto Tartari \\ Istituto di Fisica del Plasma - Consiglio Nazionale delle Ricerche \\ E-mail: wbineifp.cnr.it
}

\section{Ocleto D'Arcangelo}

ENEA for EUROfusion

\section{Cristian Galperti}

Ecole Polytéchnique Fédérale de Lausanne, Centre de Recherches en Physique des Plasmas

During the last years anomalous signals have been found in the spectra analyzed with the Collective Thomson Scattering (CTS) diagnostics in tokamak devices like TEXTOR, ASDEX and FTU. The signal frequency, down-shifted with respect to the probing one, suggested the explanation of their origin in terms of Parametric Decay Instability (PDI) processes correlated with the presence of magnetic islands and taking place even for values of the pumping wave power below the threshold predicted by conventional models. A threshold lower or comparable with the power levels routinely used could pose limitations to the use of the ECRH in fusion devices. An accurate characterization of the conditions for the occurrence of this phenomenon and of its consequences is thus of primary importance. The CTS diagnostics of FTU has been recently improved for this aim with a new fast data acquisition, allowing spectra reconstruction by direct FFT of the intermediate frequency signal, added in parallel to the pre-existing multichannel spectrum analyzer. A section with polarizing mirrors has been introduced in the quasi-optical transmission line to control the polarization of the received signals. Exploiting the front-steering configuration available with the real-time launcher of FTU, the setup allows studying the anomalous emissions in conditions of density and wave injection similar to ITER. Given the possibility of the CTS in FTU to operate in both $\mathrm{O}$ - and $\mathrm{X}$-mode, a second radiometer is being installed, to detect simultaneously signals scattered in both polarizations. The upgrades mentioned above are presented in this work as well as the first data obtained in 2014, during the experimental activity carried out under an EUROfusion Enabling Research project.

1st EPS conference on Plasma Diagnostics

14-17 April 2015

Frascati, Italy

\footnotetext{
${ }^{*}$ Speaker.

${ }^{\dagger}$ Corresponding author.
} 


\section{Introduction}

In its earlier state, the main aim of the Collective Thomson Scattering (CTS) diagnostics in FTU [1] consisted in studying the collective Thomson scattering of millimeter waves in the propagation window below the $1^{\text {st }}$ electron cyclotron (EC) harmonic resonance, in the same condition foreseen for the CTS diagnostic of fast ion distribution function in ITER. A recent aim of this experiment is to demonstrate the possibility of measuring the radiation scattered by plasma fluctuations coherent with rotating MHD magnetic islands. This phenomenon is attributed to a nonlinear process such as parametric decay instability (PDI) of EC waves occurring in case of non-monotonic plasma density profiles. According to recent theoretical models [2] such process would occur with a probing power threshold strongly lowered with respect to the one predicted by models [3], that predict thresholds for PDI appearance several orders of magnitude higher than typical gyrotron powers. Such anomalous emission have been observed for the first time with the CTS diagnostics in TEXTOR [4] and later other evidences of signals with the same nature have been found also in other tokamaks, as ASDEX and FTU. The phenomenon might affect the detection of the thermal CTS spectra, thus preventing an accurate measurements of the ion temperature, or the use of ECRH in fusion devices. FTU is the only device that offers the possibility to carry out these studies in a plasma with density similar to the ITER one in both $\mathrm{O}$ and $\mathrm{X}$ propagation modes.

\subsection{Recent upgrades of the diagnostics}

The new CTS system installed on FTU exploits a $500 \mathrm{~kW} / 140 \mathrm{GHz}$ probe beam provided by a GYCOM gyrotron. The first experiments with the upgraded diagnostics started in 2014. One of the two lines of the real-time front-steering antenna of FTU [5] was used to launch the probe wave and the second line was used to transmit the scattered signal. This second line has been designed either to inject ECH power or to receive radiation, for both CTS experiments and studies of the reverse OXB mode coupling scheme [6]. A beam switch installed in the line allows the received signal to leave the EC transmission line and to reach a pre-existing quasi-optical receiving line feeding the $140 \mathrm{GHz}$ radiometer. The connection between such quasi-optical line and the CTS antennas was created installing a low-loss oversized corrugated waveguide, with a diameter of $88.9 \mathrm{~mm}$, and a few quasi-optical (plane and curved) mirrors as matching optics. To control the polarization of the received signals and to select the proper matching of the beam polarization ellipticity to the receiving horn, a universal polarization section, composed by a couple of corrugated mirrors with $\lambda / 4$ and $\lambda / 8$ corrugation depth has been included into the radiation path, at the end of the transmission line before the front-end. Also a numerical code, capable to calculate the beam changes along the CTS line by mapping the polarization parameters from plasma back to the polarizers, has been developed in order to define the correct configuration of the polarization section, later checked also with low power measurements.

\section{First operations with the new diagnostics}

The experiments in 2014 have been carried out using for the first time two acquisition systems operating in parallel. In fact, to study the rapidly modulated PDI emissions correlated with rotating islands, a fast digitizer has been added to the pre-existing spectrum analyzer (consisting in a 32 
channels filter bank) which allows a single-sideband detection of $\pm 1.2 \mathrm{GHz}$ from the local oscillator frequency (tuned at the same frequency of the probe beam). The new digitizer is a NI PXIe-5186 capable to analyze signals in an analog bandwidth of $5 \mathrm{GHz}$ using a resolution of 8 bit. A maximum of two channels in parallel can be used, sharing a maximum sampling rate of $12.5 \mathrm{GS} / \mathrm{s}$. The digitizer allows acquiring directly the down-converted signal by sampling it after the frontend radiometer stage and storing data for numerical FFT analysis. Now the digitizer bandwidth is limited to $\sim 1.2 \mathrm{GHz}$ due to the limitation of the IF amplifiers installed in the front-end. For this reason a substitution with amplifiers capable to operate in a wider frequency range is scheduled. The two plasma scenarios exploited in 2014 had never been tested before with the front-steering launcher of FTU. Two different toroidal magnetic fields of 7.2 $\mathrm{T}$ and $4.7 \mathrm{~T}$ have been chosen, at the same plasma current of $500 \mathrm{kA}$. From the point of view of the launcher safety the main difference between these two scenarios consists in the reciprocal position of the EC harmonic layers $\left(1^{s t}\right.$ and $2^{\text {nd }}$ harmonic resonance) and the plasma-facing launching mirror used for the probe injection. The comparison of resonant and non-resonant scenarios should be helpful to better understand the impact of the PDIs on the ECH efficiency. The interception of the probe beam with the receiving line of sight has been studied for the two toroidal angles $5^{\circ}$ and $35^{\circ}$. Angles lower than $5^{\circ}$ are discarded, to prevent direct reflections of the power back into the transmission line, while angles higher than $35^{\circ}$ would be too close to the limit of the automatic protection system of the steerable mirror. The interest in studying CTS at different toroidal angles lies in the fact that when the angle between the magnetic field and the scattering vector (difference between the two wave vectors of the incident and scattered radiation) approaches $90^{\circ}$ modulations appear in the thermal CTS spectra at the ion cyclotron frequency $\omega_{c i}$ (while PDIs emissions should instead be unaffected). In order to properly prepare the operations with the antenna a careful analysis of the configurations of the two mirrors required to locate the scattering volume on the rational surfaces of the magnetic islands has been carried out with the quasi-optical beam tracing code GRAY [7] to simulate the propagation of the probe and the received beam. Typical islands in FTU are located at the rational surfaces where the safety factor q equals the ratios $m: n=2: 1$ and $m: n=3: 2$, and they may be spontaneous or induced by Neon or Argon injection. Despite the great dynamical flexibility of the FTU launcher and its capability to intersect the beams in most of the plasma volume in real-time with continuous movements in all directions, in the first experiments of 2014 the scattering volume was moved, whenever possible, only on the equatorial plane of the torus. The reason was to preserve the symmetry of the CTS configuration allowed by the launcher, which, for probe injection and CTS detection, is provided with two plasma-facing mirrors with an up-down symmetry in the poloidal plane, with respect to the equatorial plane. One of the two plasma scenarios exploited for CTS measurements has been with a toroidal field $\mathrm{B}_{\mathrm{T}}=7.2 \mathrm{~T}$. This configuration is such that the $1^{\text {st }}$ harmonic $\mathrm{EC}$ resonance (@5 T) is out of the plasma on the low field side, allowing sub-harmonic operations with a non-resonant probe frequency, as foreseen in the CTS diagnostics of ITER. The first operations at toroidal field of 7.2 T, which was expected to be the most critical for the safety of the launcher, has been successful. The $1^{\text {st }}$ harmonic resonance is, in fact, critically positioned close to the launching mirror when operating with high magnetic fields and a risk of mirror damage can arise. The same concerns may exist also in ITER, which for CTS will operate sub-harmonic. The success of the high field operations has been confirmed both during the experiments, by monitoring the plasma spectra with UV spectroscopy, to check possible release of copper from the mirror surface, and 
at the end of the operations, performing a visual inspections of the launcher which confirmed the good condition of the mirror after the experiments. The inspection has been carried out from the vacuum side of the vessel, using the inspection system described in [8]. The second scenario has been with a lower field of $4.7 \mathrm{~T}$, with the fundamental harmonic resonance in the plasma on the high field side. This scenario has the advantage of lowering the stray radiation, strongly dumped at the cyclotron harmonic layer. It is worth noting that anomalous emissions due to PDIs are expected to be considerably stronger than ECE. The scenario with $\mathrm{B}_{\mathrm{T}}=4.7 \mathrm{~T}$ was exploited for scattering measurements either crossing the probe and receiving beams in the low field side on the equatorial plane of the torus, thus forming the scattering volume in a region far from the resonant layer @ $5 \mathrm{~T}$, or intersecting them at the $1^{\text {st }}$ harmonic resonance, in this case with an asymmetric CTS configuration and scattering volume out of the equatorial plane. This last configuration is thought to study the effects of the PDI phenomenon on ECH, at the position where the beam should be absorbed. In both cases the scattering volume has been placed in correspondence of the expected $m: n=2: 1$ tearing mode location, in order to stimulate the occurrence of anomalous signals.

\section{Discussion}

In the scattering signals different kinds of activity (even as strong as the ECE emission) have been measured in the frequency range $1.2 \mathrm{GHz}$ from the probe frequency, sometimes also in conditions when the MHD island activity was not observed. Also shots without magnetic islands were planned for checking the level of signals with no disturbances due to PDIs and to compare the scenarios with and without MHD. In most cases the emissions seem not to be clearly correlated or synchronous with any magnetic probes signal. Thermal CTS emission should be excluded, also

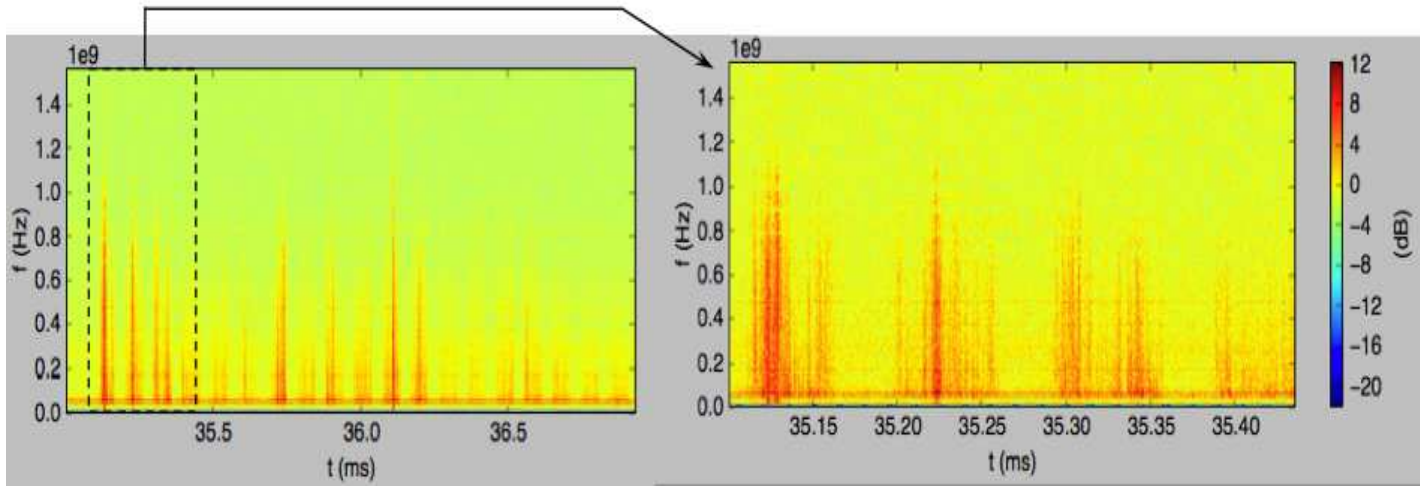

Figure 1: Left: example of spectrogram measured with the fast digitizer acquisition. Rapid emissions are detected as bursts or as spikes at frequencies in the range 30-50 kHz. Right: signals detected in the time window indicated with the dashed line in the spectrogram on the left, showing the great flexibility of the digitizer in the time resolution capability. Time on abscissa starts from the end an interval at the beginning of the pulse when the gyrotron frequency is unstable, during which a pin diode blinds the receiver to protect the mixer, while frequencies are in GHz.

with toroidal field of $7.2 \mathrm{~T}$ in absence of EC harmonic resonance in the plasma, since attenuations in this phase of experiments were too high for the detection of such a faint signal. In presence of 
more relevant MHD activity several emissions appear in the spectra in different forms: as spectral lines close to the probe frequency with a slow dynamics (if compared with the rotation time scales of the islands); as lines at higher frequencies, rapidly changing in time, in the form of rapid bursts emissions (see Figure 1); as broad-band, confined in well defined frequency ranges away from the probe frequency; as continuum. A clear correlation between signals changing rapidly in time and thermal or magnetic signals is under investigation. The formation of an undesired breakdown plasmoid in the port in front of the antenna (at the harmonic resonance layer) during the probe beam injection is also under investigation as possible origin of slow emissions of particular kind that seem to be uncorrelated with any MHD activity. Mode jumps, in fact, have been found sometimes during the operations with the gyrotron, like those shown in Figure 2-left. In some cases this led to strong signals appearing at the end of the pulse at well defined frequency distance from the probe frequency. The formation of a plasma in the antenna port may be a possible explanation of these mode jumps, due to back-reflections occurring in the transmission line. In support of this hypothesis a light was detected in several shots, as showed in Figure 2-right, using a visible-light camera looking in correspondence of the ECRH port during the pulses. The investigation and the characterization of this occurrence is important, in particular in the sub-harmonic scenario with $\mathrm{B}_{\mathrm{T}}=7.2 \mathrm{~T}$, to prevent the same phenomenon also in the CTS probe line in ITER.
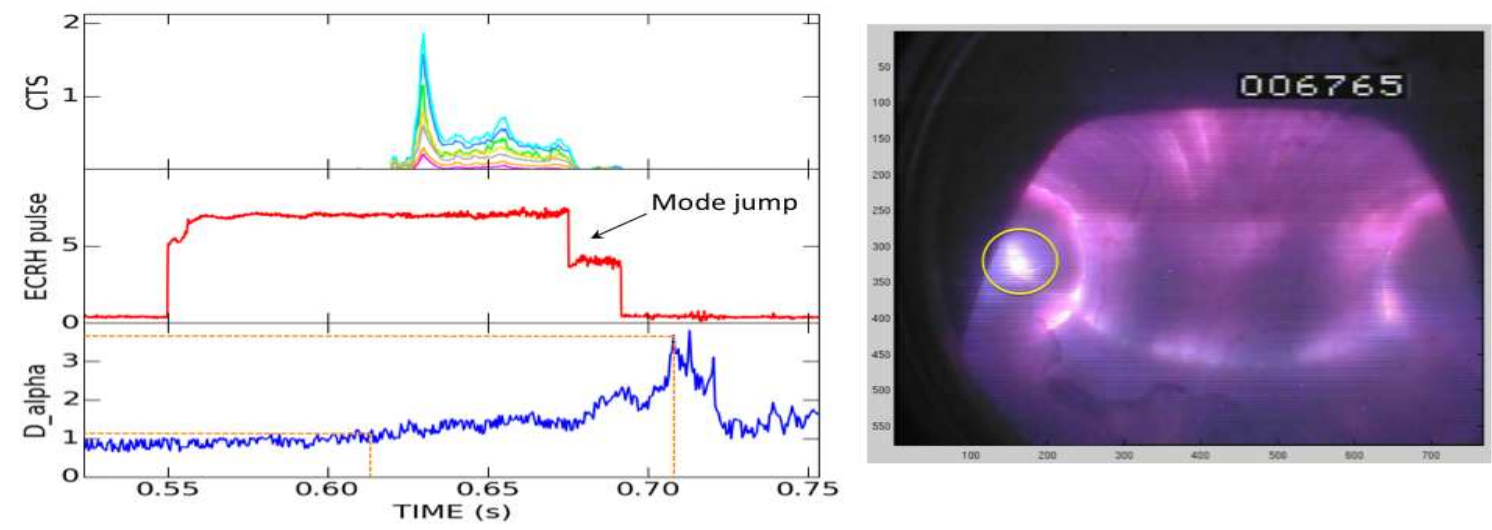

Figure 2: Left: broadband signals appearing in the CTS channels of the spectrum analyzer (shot \#38995) during the ECRH gyrotron pulse. A mode jump occurs a $\mathrm{t} \approx 670 \mathrm{~ms}$. The $\mathrm{D}_{\alpha}$ diagnostics signal gradually grows, starting from about the middle of the gyrotron pulse up to the end of it, leading to consider the formation of a breakdown plasmoid in the port the possible origin of the signals. Right: light detected during the pulse (yellow circle) with a visible-light camera looking the ECRH port from $90^{\circ}$ line of sight.

\section{Future prospects}

Apart from the mode jumps possibly due to wave reflections in the port, during the CTS experiments of 2014 the gyrotron turned out not to be stable enough in frequency to carry out accurate CTS measurements. Frequency shifts arose both during the pulse, in a range up to some tens of $\mathrm{MHz}$, and at the end of the pulse, with fast changes of about $0.5 \mathrm{GHz}$ in around $50 \mu \mathrm{s}$, making the stray gyrotron radiation fall outside the notch filter. To protect the mixer from the stray 
power, the experiments were carried out setting attenuations much higher than the optimal one (that should be $\approx 0 \mathrm{db}$ ) for CTS measurements. In addition, the same gyrotron was limited to $150 \mathrm{~ms}$ in pulse length when used for CTS, due to overheating of the collector. Therefore, in the beginning of 2015 the CTS gyrotron has been replaced with a new one which allows a longer pulse (up to $500 \mathrm{~ms}$ also for CTS) and which is stable enough in frequency. A second CTS radiometric system is also being installed in 2015, to be used in parallel to the present one. The front-end and the back-end of this system, already operating in the past in the W7-AS device, are very similar to the ones of the FTU system and now they have been reassembled. As the FTU receiver, this system is provided with 32 channels (multiplexers) and a set of bandpass filters. The new front-end and radiometer will be connected to a second branch of the polarization section of the CTS line and to the second channel of the digitizer. These upgrades will allow to perform measurements in FTU with both the channels of the digitizers (@6.25 GS/s of resolution each) and two filter banks (with 32 channels each, acquired @2 kHz) simultaneously. The two synchronized radiometric systems will be used to detect radiation in both $\mathrm{O}$ - and X-mode at the same time and to measure scattered signals from two different lines of sight. For this aim a further receiving antenna is planned in the transmission line to introduce a second line of sight for CTS, for cross-calibrations or for the gyrotron spectrum reference.

\section{Acknowledgements}

This work has been carried out within the framework of the EUROfusion Consortium and has received funding from the Euratom research and training programme 2014-2018 under grant agreement No 633053. The views and opinions expressed herein do not necessarily reflect those of the European Commission.

\section{References}

[1] U. Tartari et al., Evolution of the millimeter-wave collective Thomson scattering system of the high-field tokamak Frascati Tokamak Upgrade, Rev. Sci. Instrum. 78 (2007) 043506.

[2] E.Z. Gusakov and A.Yu. Popov, On the possibility of low-threshold anomalous absorption in tokamak 2nd-harmonic electron cyclotron resonance heating experiments, Europhys. Lett. 99 (2012) 15001.

[3] M. Porkolab and B.I. Cohen, Parametric instabilities associated with intense electron cyclotron heating in the MTX tokamak, Nucl. Fusion 28239 (1988).

[4] E. Westerhof et al., Strong Scattering of High Power Millimeter Waves in Tokamak Plasmas with Tearing Modes, Phys. Rev. Lett. 103 (2009) 125001.

[5] W. Bin et al., Design of a new ECRH launcher for FTU tokamak, Fusion Eng. Des. 84 (2009) 451.

[6] W. Bin et al., Feasibility study of $O-X$ coupling for overdense plasma heating through $O-X-B$ mode conversion in FTU, Nucl. Fusion 53 (2013) 083020.

[7] D. Farina, A quasi-optical beam-tracing code for electron cyclotron absorption and current drive: GRAY, Fusion Sci. Technol. 52 (2007) 154.

[8] W. Bin et al., Antenna system analysis and design for automatic detection and real-time tracking of electron Bernstein waves in FTU, JINST 9 (2014) P05001. 